Pacific Journal of Mathematics

ON ORTHODOX SEMIGROUPS DETERMINED BY THEIR 


\title{
ON ORTHODOX SEMIGROUPS DETERMINED BY THEIR BUNDLES OF CORRESPONDENCES
}

\author{
SIMON M. GOBERSTEIN
}

\begin{abstract}
The author's theorem that fundamental inverse semigroups are strongly determined by their bundles of correspondences is extended to the class of orthodox semigroups with fundamental maximum inverse semigroup morphic images.
\end{abstract}

0. Introduction. Let $S$ be a semigroup. A correspondence of $S$ is defined as any subsemigroup of $S \times S$ (including the empty one) or, in other words, as any stable binary relation on $S$. Let $\mathscr{C}(S)$ denote the set of all correspondences of $S$. Then $\mathscr{C}(S)$ is closed under composition (o) of binary relations and the operation $\left(^{-1}\right)$ of taking the inverse, $\rho^{-1}$, of any correspondence $\rho$ of $S$. Thus $\left(\mathscr{C}(S), \circ,{ }^{-1}\right)$ is a semigroup with an involution having a zero $\varnothing$ and an identity $\Delta_{S}$ (= the equality, or "diagonal", relation on $S$ ). Moreover, $(\mathscr{C}(S), \subseteq$ ) is an algebraic lattice and the partial order $\subseteq$ is compatible with both operations, composition and involution. Following Kurosh $[10,11]$ we say that the system $\left(\mathscr{C}(S), \circ,,^{-1}, \subseteq\right)$ is the bundle of correspondences of $S$; in what follows it will be denoted simply by $\mathscr{C}(S)$. It is obvious that $\mathscr{C}(S)=\mathscr{C}\left(S^{\text {opp }}\right)$ where $S^{\text {opp }}$ is the dual semigroup of $S$.

It ought to be mentioned that correspondences can, of course, be introduced in the general frame of category theory [14] and considered for other algebraic structures, not only for semigroups. In fact, the first (known to the author) paper on bundles of correspondences was that of Mac Lane [12] in which he studied correspondences of modules and called them "additive relations" (the author is grateful to Professor S. Mac Lane for this reference and for sending him an offprint of [12]).

Let $S$ and $S^{\prime}$ be semigroups with isomorphic bundles of correspondences. Then $S$ and $S^{\prime}$ are said to be $\mathscr{C}$-isomorphic and any isomorphism of $\mathscr{C}(S)$ onto $\mathscr{C}\left(S^{\prime}\right)$ is called a $\mathscr{C}$-isomorphism of $S$ onto $S^{\prime}$. We will say that a $\mathscr{C}$-isomorphism $\Phi$ of $S$ onto $S^{\prime}$ is induced by a bijection $\varphi$ of $S$ upon $S^{\prime}$ (or that $\varphi$ induces $\Phi$ ) if for any $\rho \in \mathscr{C}(S), \rho \Phi=\varphi^{-1} \circ \rho \circ \varphi$, that is, for all $x, y \in S,(x, y) \in \rho$ if and only if $(x \varphi, y \varphi) \in \rho \Phi$. It is obvious that any isomorphism or 
antiisomorphism of $S$ upon $S^{\prime}$ induces a $\mathscr{C}$-isomorphism of $S$ onto $S^{\prime}$. A semigroup $S$ is called $\mathscr{C}$-determined if for any semigroup $S^{\prime}$, $\mathscr{C}(S) \cong \mathscr{C}\left(S^{\prime}\right)$ if and only if $S^{\prime} \cong S$ or $S^{\prime} \cong S^{\text {opp }}$ (of course, the latter might be omitted if $S$ is antiisomorphic to itself-for example, if $S$ is commutative or inverse). We say that $S$ is strongly $\mathscr{C}$-determined if any $\mathscr{C}$-isomorphism of $S$ onto a semigroup $S^{\prime}$ is induced by an isomorphism or an antiisomorphism of $S$ upon $S^{\prime}$.

The $\mathscr{C}$-determinability of bands was established by Bredihin [1]. In [5] the author proved that fundamental inverse semigroups are strongly $\mathscr{C}$-determined. The present paper extends these results to the class of orthodox semigroups with fundamental maximum inverse semigroup morphic images. This class is properly contained in the class of fundamental orthodox semigroups (see Lemma 2.6 below and T. E. Hall's example [15, Example 1] which shows that even for the Hall semigroup $W_{E}$ of a band $E$, the maximum inverse semigroup morphic image may not be fundamental), however it forms a vast subclass of the latter. For example, it is easily seen that an orthodox semigroup is combinatorial if and only if its maximum inverse semigroup morphic image is. Thus the class of orthodox semigroups with fundamental maximum inverse semigroup morphic images contains all combinatorial orthodox semigroups (and, in particular, free orthodox *-semigroups). Many other examples of semigroups which belong to that class can be constructed using [15, Theorem 3 and Corollary 4a].

The paper is organized as follows. Section 1 contains basic information about $\mathscr{C}$-isomorphisms of arbitrary semigroups needed later. Section 2 provides preliminaries on orthodox semigroups and their $\mathscr{C}$-isomorphisms. In particular, one of the lemmas from the author's paper [5] is generalized here giving certain equivalent conditions for a bijection between an arbitrary orthodox semigroup and a fundamental one to be an isomorphism (Lemma 2.5). We use this result along with several others in $\S 3$ to prove our main theorem: if $S$ is an orthodox semigroup whose maximum inverse semigroup morphic image is fundamental, then any $\mathscr{C}$-isomorphism of $S$ onto a semigroup $S^{\prime}$ is induced by a unique bijection, and this bijection is an isomorphism or an antiisomorphism of $S$ onto $S^{\prime}$ (Theorem 3.1).

1. $\mathscr{C}$-isomorphisms of semigroups, the background. Let $S$ and $S^{\prime}$ be $\mathscr{C}$-isomorphic semigroups, and let $\Phi$ be an isomorphism of $\mathscr{C}(S)$ onto $\mathscr{C}\left(S^{\prime}\right)$. Then all properties of correspondences of $S$ expressed in terms of $\circ,-1$, and $\subseteq$ are preserved under $\Phi$. In particular, $\Delta_{S} \Phi=$ $\Delta_{S^{\prime}}$ since $\Delta_{S} \Phi$ is the identity of $\left(\mathscr{C}\left(S^{\prime}\right), \circ\right)$, and if $\varepsilon$ is a congruence 
on $S$, then $\varepsilon \Phi$ is a congruence on $S^{\prime}$. Let $\mathscr{S}_{u b}(S)$ denote the set of all subsemigroups of $S$. To indicate that $H \in \mathscr{S} u b(S)$, we write $H \leq S$. It will be assumed that $\varnothing \leq S$, so that $(\mathscr{S e b}(S), \subseteq)$ is an algebraic lattice. It is clear that if $\rho \in \mathscr{C}(S)$, then $\rho \subseteq \Delta_{S}$ if and only if $\rho=\Delta_{H}$ for some $H \leq S$. Thus $\Phi$ defines an isomorphism $\Phi^{*}$ of $\mathscr{S}_{u b}(S)$ onto $\mathscr{S}_{u b}\left(S^{\prime}\right)$ by the formula $\Delta_{H} \Phi=\Delta_{H \Phi^{*}}$ for any $H \leq S$. If $\Phi$ is induced by a bijection $\varphi: S \rightarrow S^{\prime}$, then for every $H \leq S, \Delta_{H \Phi^{*}}=\Delta_{H} \Phi=\Delta_{H \varphi}$ whence $H \Phi^{*}=H \varphi$, so that $\varphi$ induces also the lattice isomorphism $\Phi^{*}$ of $S$ onto $S^{\prime}$. It follows easily from [16] that for any $\rho \in \mathscr{C}(S)$,

$$
\operatorname{dom}(\rho \Phi)=(\operatorname{dom} \rho) \Phi^{*} \text { and } \operatorname{ran}(\rho \Phi)=(\operatorname{ran} \rho) \Phi^{*},
$$

and for any $H \leq S$,

$$
\mathscr{C}(H) \Phi=\mathscr{C}\left(H \Phi^{*}\right),
$$

that is, the restriction of $\Phi$ to $\mathscr{C}(H)$ is a $\mathscr{C}$-isomorphism of $H$ onto $H \Phi^{*}$.

Let $S$ be a semigroup. Take any $x \in S$. If $x$ has finite order, the index of $x$ is defined [9, p. 8] as the least positive integer $m$ such that $x^{m}=x^{m+n}$ for some positive integer $n$; in this case we write ind $x=m$. If $x$ has infinite order, set ind $x=\infty$. Now define ind $S=\sup \{$ ind $x: x \in S\}$. If $X$ is a nonempty subset of $S$, the subsemigroup of $S$ generated by $X$ is denoted by $\langle X\rangle$. In particular, for any $x, y \in S,\langle x\rangle$ is the monogenic subsemigroup of $S$ generated by $x$ and $\langle(x, y)\rangle$ is the monogenic subsemigroup of $S \times S$ generated by $(x, y)$.

Result 1.1 (Bredihin [2, Proposition 2.1 and its proof]; see also [5, Result 1.3 with an outline of proof]). Let $S$ and $S^{\prime}$ be $\mathscr{C}$-isomorphic semigroups, and let $\Phi$ be a $\mathscr{C}$-isomorphism of $S$ onto $S^{\prime}$. Suppose that ind $S>1$. Then $\Phi$ is induced by a unique bijection $\varphi$ of $S$ onto $S^{\prime}$. This bijection $\varphi$ can be defined by the formula

$$
\langle(a, s)\rangle \Phi=\langle(a \varphi, s \varphi)\rangle
$$

for any $s \in S$ and any $a \in S$ such that ind $a>1$.

If ind $S>2$, one can obtain more information about $\varphi$.

Result 1.2 (Bredihin [2, Lemma 2.2]). Let $\Phi$ be a $\mathscr{C}$-isomorphism of a semigroup $S$ onto a semigroup $S^{\prime}$. Suppose ind $S>2$ and let $\varphi$ be the unique bijection of $S$ onto $S^{\prime}$ inducing $\Phi$. Then for any $x, y \in$ $S$ and any integer $n \geq 1,\left(x^{n}\right) \varphi=(x \varphi)^{n}$ and $\left\{x^{2}, y^{2}, x y, y x\right\} \varphi=$ $\left\{(x \varphi)^{2},(y \varphi)^{2},(x \varphi)(y \varphi),(y \varphi)(x \varphi)\right\}$. 
Result 1.3 (the author [5, Lemma 1.5]). Let $S$ and $S^{\prime}$ be semigroups and $\Phi$ a $\mathscr{C}$-isomorphism of $S$ onto $S^{\prime}$. Let elements $x_{i}, y_{i}$ of $S(i \in I)$ and a mapping $\varphi: S \rightarrow S^{\prime}$ be such that $\left\langle\left(x_{i}, y_{i}\right)\right\rangle \Phi=$ $\left\langle\left(x_{i} \varphi, y_{i} \varphi\right)\right\rangle$ for every $i \in I$. Then

$$
\left\langle\left(x_{i}, y_{i}\right): i \in I\right\rangle \Phi=\left\langle\left(x_{i} \varphi, y_{i} \varphi\right): i \in I\right\rangle \text {. }
$$

For any semigroup $S$, denote by $E(S)$ the set of all idempotents of $S$ (of course, for some semigroups $S, E(S)$ may be empty). Now let $S$ and $S^{\prime}$ be $\mathscr{C}$-isomorphic semigroups, and let $\Phi$ be a $\mathscr{C}$-isomorphism of $S$ onto $S^{\prime}$. It follows from [16, Theorem 1.2] that $E(S)$ and $E\left(S^{\prime}\right)$ are either both empty or both nonempty and, in the latter case, there is a bijection $\varphi_{E}$ of $E(S)$ onto $E\left(S^{\prime}\right)$ defined by the formula

$$
\{(e, e)\} \Phi=\left\{\left(e \varphi_{E}, e \varphi_{E}\right)\right\}
$$

for any $e \in E(S)$. Take any $s \in S$. Since monogenic semigroups are $\mathscr{C}$-determined [1, Theorem 2.1(c)], formula (2) implies that $\langle s\rangle \Phi^{*}$ is a monogenic subsemigroup of $S^{\prime}$ isomorphic to $\langle s\rangle$. Moreover, if ind $S>1$ and if $\varphi$ is the unique bijection of $S$ onto $S^{\prime}$ which induces $\Phi$, then using formulae (1) and (3), it is easy to see that $\langle s\rangle \Phi^{*}=\langle s \varphi\rangle$.

Let $\varepsilon$ be a congruence on a semigroup $S$, and let

$$
\mathscr{C}_{\varepsilon}(S)=\{\rho \in \mathscr{C}(S): \rho \circ \varepsilon=\varepsilon \circ \rho=\rho\} .
$$

It is clear that $\left(\mathscr{C}_{\varepsilon}(S), \circ,-1, \subseteq\right)$ is a subsystem of $(\mathscr{C}(S), \circ,-1, \subseteq)$. For any $\sigma \in \mathscr{C}(S / \varepsilon)$, define

$$
\sigma \Theta_{\varepsilon}=\varepsilon^{\natural} \circ \sigma \circ\left(\varepsilon^{\natural}\right)^{-1},
$$

where $\varepsilon^{\natural}$ is the natural morphism of $S$ onto $S / \varepsilon$. Then $\Theta_{\varepsilon}$ is an isomorphism of $\mathscr{C}(S / \varepsilon)$ onto $\mathscr{C}_{\varepsilon}(S)$ (see [16, Theorem 1.7] where this fact is established for any universal algebra $S$ ).

LEMMA 1.4. Let $S$ be a semigroup, and let $\varepsilon$ be a congruence on S. Then

(i) for any $\sigma \in \mathscr{C}(S / \varepsilon)$ and any $a, b \in S,(a \varepsilon, b \varepsilon) \in \sigma$ if and only if $(a \varepsilon) \times(b \varepsilon) \subseteq \sigma \Theta_{\varepsilon}$;

(ii) for any $x, y \in S,\langle(x \varepsilon, y \varepsilon)\rangle \Theta_{\varepsilon}=\langle(x \varepsilon) \times(y \varepsilon)\rangle$.

REMARK. For the sake of clarity it should be emphasized that $\langle(x \varepsilon, y \varepsilon)\rangle$ is the monogenic subsemigroup of $(S / \varepsilon) \times(S / \varepsilon)$ generated by the pair $(x \varepsilon, y \varepsilon) \in(S / \varepsilon) \times(S / \varepsilon)$ whereas $\langle(x \varepsilon) \times(y \varepsilon)\rangle$ is the subsemigroup of $S \times S$ generated by the subset $(x \varepsilon) \times(y \varepsilon) \subseteq S \times S$. 
Proof. (i) Let $\sigma \in \mathscr{C}(S / \varepsilon)$ and $a, b \in S$. Suppose that $(a \varepsilon, b \varepsilon) \in$ $\sigma$, and take any $c \in a \varepsilon$ and $d \in b \varepsilon$. Then $c \varepsilon^{\natural}=a \varepsilon$ and $d \varepsilon^{\natural}=b \varepsilon$, so that $(c, d) \in \varepsilon^{\natural} \circ \sigma \circ\left(\varepsilon^{\natural}\right)^{-1}$. Hence, according to formula (5), $(a \varepsilon) \times(b \varepsilon) \subseteq \sigma \Theta_{\varepsilon}$.

Conversely, assume that $(a \varepsilon) \times(b \varepsilon) \subseteq \sigma \Theta_{\varepsilon}=\varepsilon^{\natural} \circ \sigma \circ\left(\varepsilon^{\natural}\right)^{-1}$. Then for some $c, d \in S$, we have $a \varepsilon^{\natural}=c \varepsilon, b \varepsilon^{\natural}=d \varepsilon$ and $(c \varepsilon, d \varepsilon) \in \sigma$. However $c \varepsilon=a \varepsilon$ and $d \varepsilon=b \varepsilon$, so that $(a \varepsilon, b \varepsilon) \in \sigma$.

(ii) Take any $x, y \in S$. It is obvious that for any integer $n \geq 1$, the $n$th power of $x \varepsilon$ in the semigroup $S / \varepsilon$ coincides with $x^{n} \varepsilon$, that is, $(x \varepsilon)^{n}=x^{n} \varepsilon$; and likewise $(y \varepsilon)^{n}=y^{n} \varepsilon$. Since $\langle(x \varepsilon, y \varepsilon)\rangle=$ $\left\{\left((x \varepsilon)^{n},(y \varepsilon)^{n}\right): n \in \mathbb{N}\right\}$, it follows from part (i) of the lemma that $\langle(x \varepsilon, y \varepsilon)\rangle \Theta_{\varepsilon}=\bigcup_{n=1}^{\infty}\left[(x \varepsilon)^{n} \times(y \varepsilon)^{n}\right]$. Therefore, $\langle(x \varepsilon, y \varepsilon)\rangle \Theta_{\varepsilon}=$ $\langle(x \varepsilon) \times(y \varepsilon)\rangle$.

Let $\Phi$ be a $\mathscr{C}$-isomorphism of a semigroup $S$ onto a semigroup $S^{\prime}$, and let $\varepsilon$ be a congruence on $S$. It is easy to see that $\Phi \mid \mathscr{C}_{\varepsilon}(S)$ is an isomorphism of $\mathscr{C}_{\varepsilon}(S)$ onto $\mathscr{C}_{\varepsilon \Phi}\left(S^{\prime}\right)$. Define

$$
\Phi_{\varepsilon}=\Theta_{\varepsilon} \circ\left(\Phi \mid \mathscr{E}_{\varepsilon}(S)\right) \circ\left(\Theta_{\varepsilon \Phi}\right)^{-1} .
$$

Since $\left(\Theta_{\varepsilon \Phi}\right)^{-1}$ is an isomorphism of $\mathscr{C}_{\varepsilon \Phi}\left(S^{\prime}\right)$ onto $\mathscr{C}\left(S^{\prime} /(\varepsilon \Phi)\right)$, the following result is immediate.

ResUlt 1.5 (from Bredihin [1, Lemma 1.1]). Let $S$ and $S^{\prime}$ be semigroups and $\Phi$ a $\mathscr{C}$-isomorphism of $S$ onto $S^{\prime}$. If $\varepsilon$ is a congruence on $S$ and if $\Phi_{\varepsilon}$ is defined by formula (6), then $\Phi_{\varepsilon}$ is a $\mathscr{C}$-isomorphism of $S / \varepsilon$ onto $S^{\prime} /(\varepsilon \Phi)$.

2. Orthodox semigroups and $\mathscr{C}$-isomorphisms, preliminaries. Recall that an orthodox semigroup is a regular semigroup in which the idempotents form a subsemigroup. In particular, bands (i.e., semigroups in which every element is idempotent) and inverse semigroups are special cases of orthodox semigroups. Therefore, in order to find out which orthodox semigroups are $\mathscr{C}$-determined, it is natural at first to consider this problem for bands and for inverse semigroups. The following two results treat these particular cases respectively and are important ingredients of the proof of our main theorem.

Result 2.1 (Bredihin [1, Theorem 2.1 (a)]). Let $E$ be a band and $E^{\prime}$ an arbitrary semigroup. If $\Phi$ is a $\mathscr{C}$-isomorphism of $E$ onto $E^{\prime}$, then $E^{\prime}$ is also a band and the mapping $\varphi_{E}$, defined by formula (4), is an isomorphism or an antiisomorphism of $E$ onto $E^{\prime}$. 
Moreover, it is easily seen that $\varphi_{E}$ induces $\Phi$ and is the only bijection of $E$ onto $E^{\prime}$ with this property. Thus bands are strongly $\mathscr{C}$-determined.

Let $S$ be a regular semigroup. Denote by $\mu_{S}$ the maximum congruence on $S$ contained in Green's relation $\mathscr{H}$ (or, which is the same [9, Proposition II.4.8], the maximum idempotent-separating congruence on $S$ ). If $\mu_{S}=\Delta_{S}, S$ is called fundamental. In [5] we established that fundamental inverse semigroups are strongly $\mathscr{C}$-determined. More precisely, the following theorem was proved.

RESUlt 2.2 (the author [5, Theorem 2.5]). Let $T$ be a fundamental inverse semigroup and $T^{\prime}$ an arbitrary semigroup. Then $\mathscr{C}(T) \cong$ $\mathscr{C}\left(T^{\prime}\right)$ if and only if $T \cong T^{\prime}$. Furthermore, any $\mathscr{C}$-isomorphism of $T$ onto $T^{\prime}$ is induced by a unique bijection, and this bijection is an isomorphism or an antiisomorphism of $T$ onto $T^{\prime}$.

Turning now to $\mathscr{C}$-isomorphisms of arbitrary orthodox semigroups, we begin with the following generalization of Result 2.1 .

LEMMA 2.3. Let $S$ be an orthodox semigroup, $S^{\prime}$ an arbitrary semigroup $\mathscr{C}$-isomorphic to $S$, and $\Phi$ a $\mathscr{C}$-isomorphism of $S$ onto $S^{\prime}$. Then $S^{\prime}$ is also orthodox and the mapping $\varphi_{E}$, defined by formula (4), is an isomorphism or an antiisomorphism of $E=E(S)$ onto $E^{\prime}=E\left(S^{\prime}\right)$.

Proof. Since $S^{\prime}$ is $\mathscr{C}$-isomorphic to $S$ and $S$ is a regular semigroup, by [2, Corollary 2.1], $S^{\prime}$ is also regular. We know that the mapping $\varphi_{E}$ given by formula (4) is a bijection of $E=E(S)$ onto $E^{\prime}=E\left(S^{\prime}\right)$. According to formula $(2), \Phi \mid \mathscr{C}(E)$ is a $\mathscr{C}$-isomorphism of $E$ onto $E \Phi^{*}$. Then, by Result 2.1,E$\Phi^{*}$ is a band and the mapping $\varphi_{E}$ is an isomorphism or an antiisomorphism of $E$ onto $E \Phi^{*}$. It remains to note that $E \Phi^{*}=\operatorname{ran} \varphi_{E}=E^{\prime}$.

If $x$ is an element of a semigroup $S$, the set of all inverses of $x$ in $S$ is denoted by $V_{S}(x)$ (or simply by $V(x)$ if there is no ambiguity about $S)$. Let $S$ be an orthodox semigroup, and let $\mathscr{Y}_{S}=\{(x, y) \in$ $\left.S \times S: V_{S}(x)=V_{S}(y)\right\}$. It is known [9, Theorem VI.1.12] that $\mathscr{Y}_{S}$ is a congruence on $S$ and that, in fact, it is the smallest inverse semigroup congruence on $S$. Thus $S / \mathscr{Y}_{S}$ is the maximum inverse semigroup morphic image of $S$.

Let $S$ be an orthodox semigroup and let $\Phi$ be a $\mathscr{C}$-isomorphism of $S$ onto a semigroup $S^{\prime}$. Then, by Lemma $2.3, S^{\prime}$ is also an orthodox 
semigroup. To simplify the notation, we will write $\mathscr{Y}$ and $\mathscr{Y}^{\prime}$ instead of $\mathscr{Y}_{S}$ and $\mathscr{Y}_{S^{\prime}}$ respectively. By Result $1.5, \Phi_{\mathscr{Y}}$ is a $\mathscr{C}$-isomorphism of $S / \mathscr{Y}$ onto $S^{\prime} /(\mathscr{Y} \Phi)$. Moreover, in the notation of this paragraph, we have

LEMMA 2.4. The image of $\mathscr{Y}$ under $\Phi$ coincides with $\mathscr{Y}^{\prime}$, so that $\Phi_{\mathscr{Y}}$ is a $\mathscr{C}$-isomorphism of $S / \mathscr{Y}$ onto $S^{\prime} / \mathscr{Y}^{\prime}$.

Proof. Since $S / \mathscr{Y}$ is an inverse semigroup, by [2, Corollary 2.2], $S^{\prime} /(\mathscr{Y} \Phi)$ is also inverse. Hence $\mathscr{Y}^{\prime} \subseteq \mathscr{Y} \Phi$. Now note that $\Phi^{-1}$ is a $\mathscr{C}$-isomorphism of $S^{\prime}$ onto $S$. Hence, by symmetry, $\mathscr{Y} \subseteq \mathscr{Y}^{\prime} \Phi^{-1}$ whence $\mathscr{Y} \Phi \subseteq \mathscr{Y}^{\prime}$. Therefore $\mathscr{Y} \Phi=\mathscr{Y}^{\prime}$.

The next lemma generalizes part of [5, Lemma 2.1]. It will play an important role in the proof of the main theorem.

LEMMA 2.5. Let $S$ and $S^{\prime}$ be orthodox semigroups and $\varphi$ a bijection of $S$ onto $S^{\prime}$ which maps $E(S)$ onto $E\left(S^{\prime}\right)$. If $S^{\prime}$ is fundamental, then the following statements are equivalent:

(i) for any $s \in S$, any $s^{\prime} \in V(s)$ and any $e \in E(S),\left(s s^{\prime}\right) \varphi=$ $(s \varphi)\left(s^{\prime} \varphi\right),(e s) \varphi=(e \varphi)(s \varphi)$ and $(s e) \varphi=(s \varphi)(e \varphi)$;

(ii) for any $s \in S$, any $s^{\prime} \in V(s)$ and any $e \in E(S), s^{\prime} \varphi \in V(s \varphi)$ and $\left(s^{\prime} e s\right) \varphi=\left(s^{\prime} \varphi\right)(e \varphi)(s \varphi)$;

(iii) $\varphi$ is an isomorphism of $S$ onto $S^{\prime}$.

Proof. (i) $\Rightarrow$ (ii). To prove this implication we do not need the assumption that $S^{\prime}$ is fundamental. Suppose that (i) holds and take any $s \in S, s^{\prime} \in V(s)$ and $e \in E(S)$. Then

$$
(s \varphi)\left(s^{\prime} \varphi\right)(s \varphi)=\left(s s^{\prime}\right) \varphi \cdot(s \varphi)=\left(s s^{\prime} s\right) \varphi=s \varphi
$$

and by symmetry $\left(s^{\prime} \varphi\right)(s \varphi)\left(s^{\prime} \varphi\right)=s^{\prime} \varphi$, so $s^{\prime} \varphi \in V(s \varphi)$. Since $s^{\prime} e \in$ $V(e s)$ [9, Theorem VI.1.1] we also have

$$
\begin{aligned}
\left(s^{\prime} e s\right) \varphi & =\left[\left(s^{\prime} e\right)(e s)\right] \varphi=\left(s^{\prime} e\right) \varphi \cdot(e s) \varphi \\
& =\left(s^{\prime} \varphi\right)(e \varphi)(e \varphi)(s \varphi)=\left(s^{\prime} \varphi\right)(e \varphi)(s \varphi) .
\end{aligned}
$$

(ii) $\Rightarrow$ (iii). Assume that (ii) holds and suppose that $S^{\prime}$ is fundamental. Take any $x, y \in S$ and any $x^{\prime} \in V(x), y^{\prime} \in V(y)$. Again by [9, Theorem VI.1.1], $y^{\prime} x^{\prime} \in V(x y)$. Therefore $\left(y^{\prime} x^{\prime}\right) \varphi \in V((x y) \varphi)$ and for each $e \in E(S)$,

$$
\begin{aligned}
& \left(y^{\prime} x^{\prime}\right) \varphi \cdot(e \varphi) \cdot(x y) \varphi=\left[\left(y^{\prime} x^{\prime}\right) e(x y)\right] \varphi=\left[y^{\prime}\left(x^{\prime} e x\right) y\right] \varphi \\
& =\left(y^{\prime} \varphi\right) \cdot\left(x^{\prime} e x\right) \varphi \cdot(y \varphi)=\left[\left(y^{\prime} \varphi\right)\left(x^{\prime} \varphi\right)\right](e \varphi)[(x \varphi)(y \varphi)] .
\end{aligned}
$$


Since $\left(y^{\prime} \varphi\right)\left(x^{\prime} \varphi\right) \in V(x \varphi \cdot y \varphi)$ and $E(S) \varphi=E\left(S^{\prime}\right)$, it follows that $((x y) \varphi,(x \varphi)(y \varphi)) \in \mu_{S^{\prime}}$ (see [9, Theorem VI.1.17]). However we assumed that $\mu_{S^{\prime}}=\Delta_{S^{\prime}}$, so that $(x y) \varphi=(x \varphi)(y \varphi)$. Hence $\varphi$ is an isomorphism of $S$ onto $S^{\prime}$.

Since (iii) trivially implies (i), the lemma is proved.

Let $S$ be an orthodox semigroup and let $T=S / \mathscr{Y}$. If $(a, b) \in \mu_{S}$, it is immediate from [9, Theorems V.3.2 and VI.1.17] that $(a \mathscr{Y}, b \mathscr{Y})$ $\in \mu_{T}$. Thus $\mu_{S} \subseteq \mathscr{Y}^{\natural} \circ \mu_{T} \circ\left(\mathscr{Y}^{\natural}\right)^{-1}$. If $\mu_{T}=\Delta_{T}$, then $\mu_{S} \subseteq$ $\mathscr{Y}^{\natural} \circ\left(\mathscr{Y}^{\natural}\right)^{-1}=\mathscr{Y}$, so by [9, formula (VI.1.16)], $\mu_{S}=\mu_{S} \cap \mathscr{Y} \subseteq \mathscr{H} \cap \mathscr{Y}$. $=\Delta_{S}$ and hence $\mu_{S}=\Delta_{S}$. We have proved

LEMMA 2.6. Let $S$ be an orthodox semigroup and $T$ its maximum inverse semigroup morphic image. If $T$ is fundamental, then $S$ is fundamental as well.

An element of a semigroup $S$ is called nongroup if it does not belong to any subgroup of $S$. It is obvious that if $S$ is an orthodox semigroup, $I$ a proper ideal of $S$ and $\rho$ a congruence on $S$ such that $x \rho y$ for some $x \in S \backslash I$ and $y \in I$, then there exist $\rho$-related idempotents $e \in D_{x}$ and $f \in I$ (e.g., for any $x^{\prime} \in V(x)$ and any $\left(y x^{\prime}\right)^{\prime} \in V\left(y x^{\prime}\right)$, we have $\left.x x^{\prime} \rho y x^{\prime}\left(y x^{\prime}\right)^{\prime} x x^{\prime}\right)$. Now as a simple corollary to [4] we obtain the following result (which could also be proved directly).

LEMMA 2.7. Let $S$ be an orthodox semigroup, $s$ an arbitrary nongroup element of $S$ and $s^{\prime} \in V(s)$. Then either $\left\{s, s^{\prime}, s s^{\prime}, s^{\prime} s\right\}$ is the top $\mathscr{D}$-class of $\left\langle s, s^{\prime}\right\rangle$ or ind $s=\infty$.

Proof. Let $F=\langle p, q\rangle$ be the free orthodox semigroup on two mutually inverse generators $p$ and $q$. Then $\left\langle s, s^{\prime}\right\rangle=F / \varepsilon$ for some congruence $\varepsilon$ on $F$. Let $\mathscr{Y}=\mathscr{Y}_{F}$ and $E=E(F)$. If $\varepsilon \subseteq \mathscr{Y}$, the free monogenic inverse semigroup $F / \mathscr{Y}$ is a morphic image of $\left\langle s, s^{\prime}\right\rangle$ and hence ind $s=\infty$. Suppose that $\varepsilon \nsubseteq \mathscr{Y}$ and recall that $s$ is a nongroup element. Then, by [4, Theorem 2.1], there exist congruences $\zeta$ and $\eta$ on $S$ such that $\zeta \subseteq \eta \subseteq \varepsilon, \zeta \vee \mathscr{Y}=\eta \vee \mathscr{Y}$, $\eta \cap(E \times E)=\varepsilon \cap(E \times E)$ and $F / \zeta$ is described by one of the following results from [4]: (a) Result 2.3, (b) Result 2.6, or (c) Result 2.5 (in fact, in cases (a) and (b), $\eta=\varepsilon$ ). In case (a), the bicyclic semigroup is a morphic image of $\left\langle s, s^{\prime}\right\rangle$, so ind $s=\infty$. In cases (b) and (c), $F / \zeta$ has the top $\mathscr{D}$-class $\{p, q, p q, q p\}$. By [3, Lemma 
$2.2]$, the congruence $\eta / \zeta$ can identify only $\mathscr{D}$-related idempotents of $F / \zeta$, and it is clear that $\varepsilon / \eta$ is an idempotent-separating congruence on $F / \eta$. In view of the remark preceding this lemma, it follows that in cases (b) and (c), $\left\{s, s^{\prime}, s s^{\prime}, s^{\prime} s\right\}$ is the top $\mathscr{D}$-class of $\left\langle s, s^{\prime}\right\rangle$.

3. The main theorem. We are ready to establish the main result of the paper.

THEOREM 3.1. Let $S$ be an orthodox semigroup whose maximum inverse semigroup morphic image is fundamental, and let $S^{\prime}$ be an arbitrary semigroup. If $S$ and $S^{\prime}$ are $\mathscr{C}$-isomorphic, then any $\mathscr{C}$ isomorphism of $S$ onto $S^{\prime}$ is induced by a unique bijection, and this bijection is an isomorphism or an antiisomorphism of $S$ onto $S^{\prime}$. In particular, $S$ is strongly $\mathscr{C}$-determined.

Proof. It will be convenient to present several stages of the argument as lemmas. They will be formulated and proved within the main proof at appropriate places without further comments. Suppose that $S$ and $S^{\prime}$ are $\mathscr{C}$-isomorphic, and let $\Phi$ be a $\mathscr{C}$-isomorphism of $S$ onto $S^{\prime}$. By Lemma 2.3, $S^{\prime}$ is an orthodox semigroup and the mapping $\varphi_{E}$, defined by formula (4), is an isomorphism or an antiisomorphism of $E=E(S)$ onto $E^{\prime}=E\left(S^{\prime}\right)$. As in $\S 2$, denote $\mathscr{Y}=\mathscr{Y}_{S}$ and $\mathscr{Y}^{\prime}=\mathscr{Y}_{S^{\prime}}$. Let $T=S / \mathscr{Y}$ and $T^{\prime}=S^{\prime} / \mathscr{Y}^{\prime}$. By Lemma $2.4, \Phi_{\mathscr{Y}}$ is a $\mathscr{C}$-isomorphism of $T$ onto $T^{\prime}$. According to the assumption, $T$ is a fundamental inverse semigroup. Suppose, first, that $T$ is a union of groups. Then $T$ is a fundamental Clifford semigroup and hence a semilattice. Take any $s \in S$. Since $(s \mathscr{Y})^{2}=s \mathscr{Y}$, by Lallement's Lemma [9, Lemma II.4.6], $s \mathscr{Y}=e \mathscr{Y}$ for some $e \in E$. Thus $s \in$ $e \mathscr{Y}=V(e)$, so by [9, Theorem VI.1.1], $s \in E$. Therefore $S=E$ and the theorem holds in this case by Result 2.1 and the remark that follows it.

Now suppose that $T$ contains nongroup elements. Let us choose a nongroup element $b$ of $T$ and fix it. Since the monogenic inverse subsemigroup $\left\langle b, b^{-1}\right\rangle$ of $T$ is not a group, it follows (see, for example [13, Chapter IX]) that either $\left\{b, b^{-1}, b b^{-1}, b^{-1} b\right\}$ is the top $\mathscr{D}$-class of $\left\langle b, b^{-1}\right\rangle$ or $\left\langle b, b^{-1}\right\rangle$ is the bicyclic semigroup; in the latter case, without loss of generality, we may assume that $b b^{-1}>b^{-1} b$ (so that $b b^{-1}$ is the identity element of $\left\langle b, b^{-1}\right\rangle$ ). Let us also choose and fix an arbitrary element $a \in S$ such that $a \mathscr{Y}=b$. Since $\mathscr{Y}^{\natural}$ is a morphism of $S$ upon $T$ and $b$ is a nongroup element of $T$, we have 
that $a$ is a nongroup element of $S$. It is clear that ind $a>1$ and ind $b>1$. By Result 1.1, there exists a unique bijection $\varphi: S \rightarrow S^{\prime}$ inducing $\Phi$, and there exists a unique bijection $\varphi_{\mathscr{y}}: T \rightarrow T^{\prime}$ which induces $\Phi_{y} ; \varphi$ is given by formula (3) and $\varphi_{y}$ by the similar formula

$$
\langle(b, t)\rangle \Phi_{\mathscr{y}}=\left\langle\left(b \varphi_{\mathscr{Y}}, t \varphi_{\mathscr{y}}\right)\right\rangle
$$

for any $t \in T$. Since $T$ is fundamental, by Result 2.2, $\varphi_{\mathscr{Y}}$ is an isomorphism or an antiisomorphism of $T$ onto $T^{\prime}$.

Case I. Assume that $\varphi_{\mathscr{y}}$ is an isomorphism of $T$ onto $T^{\prime}$.

LEMMA 3.2. The restriction of $\varphi$ to $E$ coincides with $\varphi_{E}$ and hence is an isomorphism or an antiisomorphism of $E$ onto $E^{\prime}$.

Proof. Take any $e \in E$. Since $\langle(a, e)\rangle \circ\{(e, e)\}=\langle(a, e)\rangle$, we have $\langle(a, e)\rangle \Phi \circ\{(e, e)\} \Phi=\langle(a, e)\rangle \Phi$, so $\langle(a \varphi, e \varphi)\rangle \circ\left\{\left(e \varphi_{E}, e \varphi_{E}\right)\right\}=$ $\langle(a \varphi, e \varphi)\rangle$ and therefore $e \varphi=e \varphi_{E}$. Thus $\varphi \mid E=\varphi_{E}$.

LeMma 3.3. For any $s \in S,(s \mathscr{Y}) \varphi_{\mathscr{Y}}=(s \varphi) \mathscr{Y}^{\prime}$.

Proof. Take any $s \in S$ and set $s \mathscr{Y}=t$. Using formula (7) and Lemma 1.4(ii), we find that

$$
\langle(b, t)\rangle\left(\Phi_{\mathscr{y}} \circ \Theta_{\mathscr{y}^{\prime}}\right)=\left\langle\left(b \varphi_{\mathscr{y}}, t \varphi_{\mathscr{y}}\right)\right\rangle \Theta_{\mathscr{y}^{\prime}}=\left\langle\left(b \varphi_{\mathscr{y}}\right) \times\left(t \varphi_{\mathscr{y}}\right)\right\rangle .
$$

On the other hand, applying Lemma 1.4(ii) again, we get

$$
\langle(b, t)\rangle\left[\Theta_{\mathscr{y}} \circ\left(\Phi \mid \mathscr{C}_{\mathscr{y}}(S)\right)\right]=\langle b \times t\rangle \Phi \text {. }
$$

Therefore, by formula (6) and Lemma $2.4,\langle b \times t\rangle \Phi=\left\langle\left(b \varphi_{\mathscr{y}}\right) \times\left(t \varphi_{\mathscr{y}}\right)\right\rangle$, that is, $\langle(a \mathscr{Y}) \times(s \mathscr{Y})\rangle \Phi=\left\langle(a \mathscr{Y}) \varphi_{\mathscr{Y}} \times(s \mathscr{Y}) \varphi_{\mathscr{Y}}\right\rangle$.

Since $\varphi$ induces $\Phi$, using Result 1.3 and Lemma 2.4, we obtain

$$
\begin{aligned}
\langle(a \mathscr{Y}) \times(s \mathscr{Y})\rangle \Phi & =\langle(x, y): x \in a \mathscr{Y}, y \in s \mathscr{Y}\rangle \Phi \\
& =\left\langle(x \varphi, y \varphi): x \varphi \in(a \varphi) \mathscr{Y}^{\prime}, y \varphi \in(s \varphi) \mathscr{Y}^{\prime}\right\rangle \\
& =\left\langle(a \varphi) \mathscr{Y}^{\prime} \times(s \varphi) \mathscr{Y}^{\prime}\right\rangle .
\end{aligned}
$$

Thus $\left\langle(a \mathscr{Y}) \varphi_{\mathscr{Y}} \times(s \mathscr{Y}) \varphi_{\mathscr{Y}}\right\rangle=\left\langle(a \varphi) \mathscr{Y}^{\prime} \times(s \varphi) \mathscr{Y}^{\prime}\right\rangle$. Applying Lemma 1.4(ii) and recalling that $\Theta_{\mathscr{Y}^{\prime}}$ is an isomorphism of $\mathscr{C}\left(S^{\prime} / \mathscr{Y}^{\prime}\right)$ onto $\mathscr{C}_{\mathscr{Y}^{\prime}}\left(S^{\prime}\right)$, we get $\left\langle\left((a \mathscr{Y}) \varphi_{\mathscr{y}},(s \mathscr{Y}) \varphi_{\mathscr{Y}}\right)\right\rangle=\left\langle\left((a \varphi) \mathscr{Y}^{\prime},(s \varphi) \mathscr{Y}^{\prime}\right)\right\rangle$. Since $a \mathscr{Y}$ is a nongroup element of $T$ and $\varphi_{y}$ is an isomorphism of $T$ onto $T^{\prime},(a \mathscr{Y}) \varphi_{y}$ is a nongroup element of $T^{\prime}$. Then it is easily seen that $\left((a \mathscr{Y}) \varphi_{\mathscr{y}},(s \mathscr{Y}) \varphi_{\mathscr{Y}}\right)=\left((a \varphi) \mathscr{Y}^{\prime},(s \varphi) \mathscr{Y}^{\prime}\right)$, and so $(s \mathscr{Y}) \varphi_{\mathscr{Y}}=$ $(s \varphi) \mathscr{Y}^{\prime}$. 
LeMma 3.4. For any $s \in S$ and any $s^{\prime} \in V(s), s^{\prime} \varphi \in V(s \varphi)$.

Proof. Take any $s \in S$ and $s^{\prime} \in V(s)$. Using Lemma 3.3 and the fact that $\varphi_{y}$ is an isomorphism of $T$ onto $T^{\prime}$, we obtain

$$
\begin{aligned}
(s \varphi) \mathscr{Y}^{\prime} \cdot\left(s^{\prime} \varphi\right) \mathscr{Y}^{\prime} \cdot(s \varphi) \mathscr{Y}^{\prime} & =(s \mathscr{Y}) \varphi_{\mathscr{Y}} \cdot\left(s^{\prime} \mathscr{Y}\right) \varphi_{\mathscr{Y}} \cdot(s \mathscr{Y}) \varphi_{\mathscr{Y}} \\
& =\left[(s \mathscr{Y})\left(s^{\prime} \mathscr{Y}\right)(s \mathscr{Y})\right] \varphi_{\mathscr{Y}}=\left[\left(s s^{\prime} s\right) \mathscr{Y}\right] \varphi_{\mathscr{Y}} \\
& =(s \mathscr{Y}) \varphi_{\mathscr{Y}}=(s \varphi) \mathscr{Y}^{\prime},
\end{aligned}
$$

and by symmetry $\left(s^{\prime} \varphi\right) \mathscr{Y}^{\prime} \cdot(s \varphi) \mathscr{Y}^{\prime} \cdot\left(s^{\prime} \varphi\right) \mathscr{Y}^{\prime}=\left(s^{\prime} \varphi\right) \mathscr{Y}^{\prime}$. Hence $\left(s^{\prime} \varphi\right) \mathscr{Y}^{\prime}$ $=\left[(s \varphi) \mathscr{Y}^{\prime}\right]^{-1}=\left((s \varphi)^{\prime}\right) \mathscr{Y}^{\prime}$ for some $(s \varphi)^{\prime} \in V(s \varphi)$. Since $\left((s \varphi)^{\prime}\right) \mathscr{Y}^{\prime}$ $=V(s \varphi)\left[9\right.$, formula (VI.1.13)], we have $s^{\prime} \varphi \in V(s \varphi)$.

LEMMA 3.5. For any $s_{1}, s_{2} \in S, V\left(\left(s_{1} s_{2}\right) \varphi\right)=V\left(\left(s_{1} \varphi\right)\left(s_{2} \varphi\right)\right)$.

Proof. Take any $s_{1}, s_{2} \in S$. Since $\varphi_{\mathscr{Y}}$ is an isomorphism of $T$ onto $T^{\prime}$, applying Lemma 3.3, we have

$$
\begin{aligned}
{\left[\left(s_{1} s_{2}\right) \varphi\right] \mathscr{Y}^{\prime} } & =\left[\left(s_{1} s_{2}\right) \mathscr{Y}\right] \varphi_{\mathscr{Y}}=\left[\left(s_{1} \mathscr{Y}\right)\left(s_{2} \mathscr{Y}\right)\right] \varphi_{\mathscr{Y}} \\
& =\left(s_{1} \mathscr{Y}\right) \varphi_{\mathscr{Y}} \cdot\left(s_{2} \mathscr{Y}\right) \varphi_{\mathscr{Y}}=\left(s_{1} \varphi\right) \mathscr{Y}^{\prime} \cdot\left(s_{2} \varphi\right) \mathscr{Y}^{\prime} \\
& =\left[\left(s_{1} \varphi\right)\left(s_{2} \varphi\right)\right] \mathscr{Y}^{\prime} .
\end{aligned}
$$

Therefore $V\left(\left(s_{1} s_{2}\right) \varphi\right)=V\left(\left(s_{1} \varphi\right)\left(s_{2} \varphi\right)\right)$.

LEMMA 3.6. For any nongroup element $s$ of $S$ and any $s^{\prime} \in V(s)$, $\left(s s^{\prime}\right) \varphi=(s \varphi)\left(s^{\prime} \varphi\right)$.

Proof. Let $s$ be an arbitrary nongroup element of $S$. It is easily shown (see, for example, [16, Theorem 2.6]) that if $H$ is a subgroup of $S$, then $H \Phi^{*}(=H \varphi)$ is a subgroup of $S^{\prime}$. Therefore $s \varphi$ is a nongroup element of $S^{\prime}$. Take any $s^{\prime} \in V(s)$ and note that

$$
\begin{aligned}
\left\langle s, s^{\prime}\right\rangle \Phi^{*} & =\left(\langle s\rangle \vee\left\langle s^{\prime}\right\rangle\right) \Phi^{*}=\langle s\rangle \Phi^{*} \vee\left\langle s^{\prime}\right\rangle \Phi^{*} \\
& =\langle s \varphi\rangle \vee\left\langle s^{\prime} \varphi\right\rangle=\left\langle s \varphi, s^{\prime} \varphi\right\rangle .
\end{aligned}
$$

By Lemma 2.7 we have that either $\left\{s, s^{\prime}, s s^{\prime}, s^{\prime} s\right\}$ is the top $\mathscr{D}$-class of $\left\langle s, s^{\prime}\right\rangle$ or ind $s=\infty$. In the first case, $\left\{s s^{\prime}, s^{\prime} s\right\}$ and $\left\{(s \varphi)\left(s^{\prime} \varphi\right)\right.$, $\left.\left(s^{\prime} \varphi\right)(s \varphi)\right\}$ are the sets of maximal idempotents of semigroups $\left\langle s, s^{\prime}\right\rangle$ and $\left\langle s \varphi, s^{\prime} \varphi\right\rangle$, respectively, and therefore, by Lemma 3.2, $\left\{s s^{\prime}, s^{\prime} s\right\} \varphi=\left\{(s \varphi)\left(s^{\prime} \varphi\right),\left(s^{\prime} \varphi\right)(s \varphi)\right\}$. If ind $s=\infty$, then, by Result 1.2, $\left\{s^{2},\left(s^{\prime}\right)^{2}, s s^{\prime}, s^{\prime} s\right\} \varphi=\left\{(s \varphi)^{2},\left(s^{\prime} \varphi\right)^{2},(s \varphi)\left(s^{\prime} \varphi\right),\left(s^{\prime} \varphi\right)(s \varphi)\right\}$, $\left(s^{2}\right) \varphi=(s \varphi)^{2}$ and $\left[\left(s^{\prime}\right)^{2}\right] \varphi=\left(s^{\prime} \varphi\right)^{2}$, whence $\left\{s s^{\prime}, s^{\prime} s\right\} \varphi=\left\{(s \varphi)\left(s^{\prime} \varphi\right)\right.$, $\left.\left(s^{\prime} \varphi\right)(s \varphi)\right\}$ again. 
Suppose that $\left(s s^{\prime}\right) \varphi=\left(s^{\prime} \varphi\right)(s \varphi)$. By Lemma 3.5, $V\left(\left(s s^{\prime}\right) \varphi\right)=$ $V\left((s \varphi)\left(s^{\prime} \varphi\right)\right)$. Denote $x=s \varphi$ and $x^{\prime}=s^{\prime} \varphi$. Then $V\left(x x^{\prime}\right)=V\left(x^{\prime} x\right)$ and by Lemma 3.4, $x^{\prime} \in V(x)$. It follows that $x^{\prime} x \in V\left(x x^{\prime}\right)$ and therefore, by [9, Theorem II.3.5 (i)], $H_{x}$ and $H_{x^{\prime}}$ contain idempotents. However this contradicts the fact that $x=s \varphi$ is a nongroup element. Therefore $\left(s s^{\prime}\right) \varphi=(s \varphi)\left(s^{\prime} \varphi\right)$.

LEMMA 3.7. For any $s \in S$ and any $e \in E,(e s) \varphi=(e \varphi)(s \varphi)$ and $(s e) \varphi=(s \varphi)(e \varphi)$. In particular, $\varphi \mid E$ is an isomorphism of $E$ onto $E^{\prime}$.

Proof. From the way the element $b \in T$ was chosen, it follows that the only possibility to get $b^{-1}$ as the result of some product of $b^{-1} b$ and $b^{-1}$ is to take $\left(b^{-1} b\right)^{n} b^{-1}$ for some $n \geq 0$ (see [5, the proof of Theorem 2.5, p. 482] with $b$ in place of $a$ there). Let $a^{\prime}$ be an arbitrary inverse of $a$. Since $\mathscr{Y}^{\mathrm{h}}$ is a morphism of $S$ onto $T$ and $a \mathscr{Y}^{\natural}=b$, we conclude that the only way to get $a^{\prime}$ as the result of some product of $a^{\prime} a$ and $a^{\prime}$ is to take $\left(a^{\prime} a\right)^{n} a^{\prime}, n \geq 0$. Choose any $s \in S$ and any $e \in E$. Let

$$
\zeta=\left\langle\left(a^{\prime} a, e\right),\left(a^{\prime}, s\right)\right\rangle \text {. }
$$

From the above remark, it follows that $a^{\prime} \zeta=\{s$,es $\}$. Using Result 1.3 , we get

$$
\zeta \Phi=\left\langle\left(\left(a^{\prime} a\right) \varphi, e \varphi\right),\left(a^{\prime} \varphi, s \varphi\right)\right\rangle .
$$

Now recall that $\varphi_{y}$ is an isomorphism of $T$ onto $T^{\prime}$ and that $b \varphi_{y}=(a \varphi)\left(\mathscr{Y}^{\prime}\right)^{\natural}$ by Lemma 3.3, $a^{\prime} \varphi \in V(a \varphi)$ by Lemma 3.4, and $\left(a^{\prime} a\right) \varphi=\left(a^{\prime} \varphi\right)(a \varphi)$ by Lemma 3.6. Therefore, similarly to the above, $\left(a^{\prime} \varphi\right)(\zeta \Phi)=\{s \varphi,(e \varphi)(s \varphi)\}$. Since $\varphi$ induces $\Phi$, it follows that $\{s, e s\} \varphi=\{s \varphi,(e \varphi)(s \varphi)\}$ whence $(e s) \varphi=(e \varphi)(s \varphi)$. Finally, note that $\Phi$ is also a $\mathscr{C}$-isomorphism of $S^{\text {opp }}$ onto $\left(S^{\prime}\right)^{\text {opp }}$ induced by the same unique bijection $\varphi$ and that $a^{\prime} \in V_{S^{\text {op }}}(a)$, $a^{\prime} \varphi \in V_{\left(S^{\prime}\right)^{\mathrm{opp}}}(a \varphi)$ and $\left(a^{\prime} * a\right) \varphi=\left(a^{\prime} \varphi\right) *(a \varphi)$ where $*$ denotes multiplication in $S^{\text {opp }}$ and $\left(S^{\prime}\right)^{\text {opp }}$. Therefore, dually, $(e * s) \varphi=(e \varphi) *(s \varphi)$, that is, $(s e) \varphi=(s \varphi)(e \varphi)$.

LEMMA 3.8. For any $s \in S$ and any $s^{\prime} \in V(s),\left(s s^{\prime}\right) \varphi=(s \varphi)\left(s^{\prime} \varphi\right)$.

Proof. Take any $s \in S$ and any $s^{\prime} \in V(s)$. By Lemma 3.6, we have to consider only the case when $s$ belongs to a certain subgroup of $S$. Thus suppose that the $\mathscr{H}$-class $H_{s}$ is a group. If $s^{\prime} \in H_{s}$, then $s^{\prime} \varphi \in H_{s} \Phi^{*}=H_{s} \varphi$ and therefore $\left(s s^{\prime}\right) \varphi=(s \varphi)\left(s^{\prime} \varphi\right)$. Now assume 
that $s^{\prime} \notin H_{s}$. Since $S$ is orthodox, $H_{s^{\prime}}$ is also a group. Let $e$ be the identity of $H_{s}$ and $f$ the identity of $H_{s^{\prime}}$. By [9, Theorem II.3.5 (i)], $\left(e, s s^{\prime}\right) \in \mathscr{R}$ and $\left(f, s s^{\prime}\right) \in \mathscr{L}$. According to Lemma 3.7, $\varphi \mid E$ is an isomorphism of $E$ onto $E^{\prime}$. Therefore $\left(e \varphi,\left(s s^{\prime}\right) \varphi\right) \in \mathscr{R}$ and $\left(f \varphi,\left(s s^{\prime}\right) \varphi\right) \in \mathscr{L}$. Since $H_{e \varphi}=H_{s \varphi}$ and $H_{f \varphi}=H_{s^{\prime} \varphi}$, again by [9, Theorem II.3.5 (i)], $(s \varphi)\left(s^{\prime} \varphi\right) \in R_{e \varphi} \cap L_{f \varphi}$. However $R_{e \varphi} \cap L_{f \varphi}$ contains only one idempotent. Hence $\left(s s^{\prime}\right) \varphi=(s \varphi)\left(s^{\prime} \varphi\right)$ and the lemma is proved.

Now take any $s \in S$, any $s^{\prime} \in V(s)$ and any $e \in E$. Since $T^{\prime}$, being isomorphic to $T$, is fundamental, according to Lemma 2.6, $S^{\prime}$ is also fundamental. By Lemma 3.8, $\left(s s^{\prime}\right) \varphi=(s \varphi)\left(s^{\prime} \varphi\right)$, and by Lemma 3.7, $(e s) \varphi=(e \varphi)(s \varphi)$ and $(s e) \varphi=(s \varphi)(e \varphi)$. Therefore, according to Lemma $2.5, \varphi$ is an isomorphism of $S$ onto $S^{\prime}$.

Case II. Now assume that $\varphi_{\mathscr{Y}}$ is an antiisomorphism of $T$ onto
$T^{\prime}$.

Recall that $\Phi$, being a $\mathscr{C}$-isomorphism of $S$ onto $S^{\prime}$, is also a $\mathscr{C}$-isomorphism of $S^{\text {opp }}$ onto $S^{\prime}$ induced by the same unique bijection $\varphi$. It is clear that $\mathscr{Y}_{S^{\text {op }}}=\mathscr{Y}_{S}$ and $S^{\text {opp }} / \mathscr{Y}=T^{\text {opp }}$. Since $\varphi_{y}$ is an isomorphism of $T^{\text {opp }}$ onto $T^{\prime}$, from what was proved in Case I it follows that $\varphi$ is an isomorphism of $S^{\text {opp }}$ onto $S^{\prime}$, that is, an antiisomorphism of $S$ onto $S^{\prime}$. This completes the proof of the theorem.

Acknowledgment. The main result of this paper was reported at the Monash Conference on Semigroup Theory in Honour of G. B. Preston, held on July 12-14, 1990, and [6] contains a detailed outline of my original proof which used T. E. Hall's structure theorem for orthodox semigroups [7] and his solution of the isomorphism problem for HallYamada semigroups [8]. I am grateful to Dr. T. E. Hall for several useful comments on that first proof. They actually stimulated me in finding a different proof of the main theorem presented in this paper.

\section{REFERENCES}

[1] D. A. Bredihin, Involuted semigroups of stable binary relations, in Studies in Algebra, No. 4, pp. 3-12, Saratov Univ. Press, Saratov, USSR, 1974 (in Russian).

[2] _ Bundles of correspondences of semigroups, in Contemporary Algebra, No. 4, pp. 31-47, Leningrad Ped. Inst. Press, Leningrad, USSR, 1976 (in Russian).

[3] C. Eberhart and W. Williams, Congruences on an orthodox semigroup via the minimum inverse semigroup congruence, Glasgow Math. J., 18 (1977), 181-192. 
[4] _ Elementary orthodox semigroups, Semigroup Forum, 29 (1984), 351-364.

[5] S. M. Goberstein, Inverse semigroups determined by their bundles of correspondences, J. Algebra, 125 (1989), 474-488.

[6] _- Bundles of correspondences of orthodox semigroups, "Monash Conference on Semigroup Theory in Honour of G. B. Preston", T. E. Hall, P. R. Jones and J. C. Meakin, editors, World Scientific, Singapore-New Jersey-LondonHong Kong, 1991, 77-85.

[7] T. E. Hall, Orthodox semigroups, Pacific J. Math., 39 (1971), 677-686.

[8] _ The isomorphism problem for orthodox semigroups, Pacific J. Math., 136 (1989), 123-128.

[9] J. M. Howie, An Introduction to Semigroup Theory, Academic Press, LondonNew York-San Francisco, 1976.

[10] A. G. Kurosh, The Theory of Groups, 3rd ed., "Nauka", Moscow, 1967 (in Russian).

[11] _ General Algebra (Lectures of the 1969/1970 Academic Year), "Nauka", Moscow, 1974 (in Russian).

[12] S. Mac Lane, An algebra of additive relations, Proc. Nat. Acad. Sci. U.S.A., 47 (1961), 1043-1051.

[13] M. Petrich, Inverse Semigroups, Wiley, New York, 1984.

[14] D. Puppe, Korrespondenzen in Abelschen Kategorien, Math. Ann., 148 1962), $1-30$.

[15] M. B. Szendrei, Correction and supplement to "On a pull-back diagram for orthodox semigroups", Semigroup Forum, 25 (1982), 311-324.

[16] G. I. Žitomirskiǐ, Stable binary relations on universal algebras, Mat. Sb., 82 (1970), 163-174 (in Russian).

Received August 13, 1990 and in revised form March 22, 1991.

California State University

CHICO, CA 95929-0525 


\section{PACIFIC JOURNAL OF MATHEMATICS EDITORS}

V. S. VARADARAJAN

(Managing Editor)

University of California

Los Angeles, CA 90024-1555-05

Herbert Clemens

University of Utah

Salt Lake City, UT 84112

F. Michael Christ

University of California

Los Angeles, CA 90024-1555

THOMAS ENRIGHT

University of California, San Diego

La Jolla, CA 92093

\section{C. MOORE}

University of California

Berkeley, CA 94720

MARTIN SCHARLEMANN

University of California

Santa Barbara, CA 93106

HAROLD STARK

University of California, San Diego

La Jolla, CA 92093

STEVEN KERCKHOFF

Stanford University

Stanford, CA 94305

ASSOCIATE EDITORS

R. ARENS

E. F. BECKENBACH (1906-1982)

B. H. NeumanN

F. WOLF

(1904-1989)

K. YosHIDA

SUPPORTING INSTITUTIONS

UNIVERSITY OF ARIZONA

UNIVERSITY OF OREGON

UNIVERSITY OF BRITISH COLUMBIA

UNIVERSITY OF SOUTHERN CALIFORNIA

CALIFORNIA INSTITUTE OF TECHNOLOGY

UNIVERSITY OF CALIFORNIA

MONTANA STATE UNIVERSITY

STANFORD UNIVERSITY

UNIVERSITY OF NEVADA, RENO

UNIVERSITY OF HAWAII

NEW MEXICO STATE UNIVERSITY

OREGON STATE UNIVERSITY

UNIVERSITY OF UTAH

WASHINGTON STATE UNIVERSITY

UNIVERSITY OF WASHINGTON 


\section{Pacific Journal of Mathematics}

\section{Vol. 153, No. $1 \quad$ March, 1992}

Patrick Robert Ahern and Carmen Cascante, Exceptional sets for Poisson integrals of potentials on the unit sphere in $\mathbf{C}^{n}, p \leq 1 \ldots \ldots \ldots \ldots \ldots$

David Peter Blecher, The standard dual of an operator space ........... 15

Patrick Gilmer, Real algebraic curves and link cobordism .............. 31

Simon M. Goberstein, On orthodox semigroups determined by their

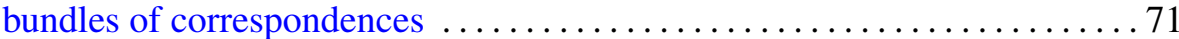

John Kalliongis and Darryl John McCullough, Homeotopy groups of irreducible 3-manifolds which may contain two-sided projective planes .......................................... 85

Yuji Konishi, Masaru Nagisa and Yasuo Watatani, Some remarks on actions of compact matrix quantum groups on $C^{*}$-algebras $\ldots \ldots \ldots \ldots 119$

Guojun Liao and Luen-Fai Tam, On the heat equation for harmonic maps from noncompact manifolds ........................... 129

John Marafino, Boundary behavior of a conformal mapping . .......... 147

Ji Min, A remark on the symmetry of solutions to nonlinear elliptic

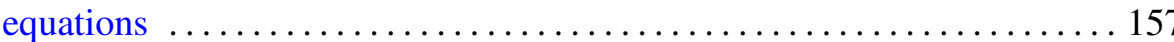

Paul Nevai and Walter Van Assche, Compact perturbations of orthogonal

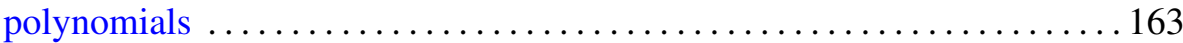

Kyril Tintarev, Level set maxima and quasilinear elliptic problems ....... 185 